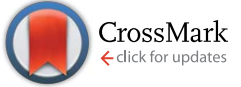

Cite this: RSC Adv., 2017, 7, 12283

Received 12th January 2017

Accepted 15th February 2017

DOI: $10.1039 / \mathrm{c} 7 \mathrm{ra00499k}$

rsc.li/rsc-advances

\section{Polydopamine-mediated preparation of an enzyme-immobilized microreactor for the rapid production of wax ester}

\begin{abstract}
Yicheng Bi, Hua Zhou, * Honghua Jia and Ping Wei
In our study, a polytetrafluoroethylene (PTFE) open-tubular enzyme-immobilized microreactor has been successfully prepared using dopamine polymerization and multi-layer deposition. The polydopamine layer was utilized as a primer layer in the microchannel to provide PTFE surface reactable groups, and polyethylenimine and Candida antarctica lipase B were alternatively adsorbed on the PTFE surface by the layer-by-layer method. The lipase loading kept increasing as the number of layers increased and reached a plateau at the 8th layer. The formation of multilayers and increase of enzyme loading were described and explained by the water contact angle measurement, Fourier transform infrared spectroscopy (FI-IR), and X-ray photoelectron spectroscopy, while the morphological characteristics were observed using Atomic Force Microscopy (AFM) and field emission scanning electron microscopy (FE-SEM). Preparation of a wax ester using the microreactor had much better production efficiency (35 minutes to reach $95 \%$ yield) than that using traditional reactors (over 4 hours to complete the reaction). Very excellent stability can be achieved by the microreactor, which could be used for 144 hours with the residual activity remaining at $83 \%$ of the initial activity.
\end{abstract}

\section{Introduction}

Due to its many advantages over conventional chemical catalysts, including less waste, mild conditions, high selectivity, less by-products, enzymes have been widely used in various fields, such as industrial biosynthesis, healthcare industry, food industry, and waste treatment. ${ }^{1}$ On the other hand, enzymes cannot be used efficiently on a large scale due to two major problems: low catalytic efficiency caused by the slow mass transfer between enzymes and substrates, and the instability of their structures under unfavorable conditions. ${ }^{2}$

Enzyme-immobilized microreactors (EI-MRs) have been a very important tool to develop the enzyme process, ${ }^{3}$ and it has been widely studied. Large surface areas give the microreactors rapid mass transfer and heat exchange, which lead to much better yield and higher production efficiency in biocatalysis., ${ }^{4,5} \mathrm{~A}$ higher conversion rate was found by Kawakami in his study ${ }^{6}$ by performing the transesterification between glycidol and vinyl $n$ butyrate in a monolithic microreactor, rather than a batch reactor. It was confirmed in $\mathrm{He}^{\prime}$ s test ${ }^{7}$ that much lower apparent Michaelis (3.1 mM of versus $38 \mathrm{mM}$ of free lipase) constant and higher conversion can be achieved with the monolithic EI-MR used for the hydrolysis of 4-nitrophenyl butyrate in two-phase media. Packed-bed microreactors were also developed and used in polymerization of end-functionalized polymers. ${ }^{\mathbf{3}}$

School of Pharmaceutical Science, Nanjing Tech University, Nanjing 211816, China. E-mail: zhouhua@njtech.edu.cn; Fax: +86-25-5813-9916; Tel: +86-25-5813-9916
However, it is well known that intricate techniques and specific instrument are required to fabricate the micro-device and modify the micro-channel surface. ${ }^{9-12}$ Fabrication of monolithic microreactors and packed-bed microreactors requires complicaused by increasing pressure. Methods to prepare opentubular micro-reactors by immobilizing enzyme directly on the micro-channel surface have also been developed by many researches ${ }^{\mathbf{1 3}, \mathbf{1 4}}$ using physical adsorption, affinity adsorption and covalent crosslinking. However, without network microstructure or packed supports, open-tube microreactors face the problem of low enzyme loading because of lower specific inner surface, which is closely related to the reaction efficiency. ${ }^{\mathbf{6}, 12,15}$ Hence it is crucial to develop a method of facile fabrication of microreactors and simplifying the enzyme immobilization step in microstructure.

PTFE microchannel has already been a commercialized product, but its surface functionalization is expensive and time consuming. The hydrophobicity and poor biocompatibility of PTFE also pose the problem for immobilization of biomolecules. Various methods used currently including transferred atmospheric pressure plasma, ${ }^{16}$ ion irradiation ${ }^{17,18}$ require expensive equipment and complex processed. Inspired by adhesive proteins excreted by marine mussels, 3,4-dihydroxyphenylalanine (DOPA) and dopamine have attracted attention for forming adhesive interaction with wide spectrum of materials. Dopamine aqueous solution will self-polymerize under the mild alkaline condition and form polydopamine (PDA) cated steps, having scale-up concerns and plugging problems 
layer, which will not only strongly adhere to various substrates, but also provide a versatile platform for secondary reactions. The PDA layer carries negative charges under alkaline conditions, which could adsorb positive materials. ${ }^{19}$ The technique is convenient and useful in bridging the gap between hydrophobic/hydrophilic materials. ${ }^{20}$ The PDA layer has been utilized to modified silica capillary to prepare electrochromatographic column, ${ }^{21}$ validating the possibility of dopamine oxidizing in microchannel only using the oxygen dissolved in solution.

In our study, PTFE microchannel, a more inert material, was chosen and tested on whether dopamine will experience polymerization in the inner surface. Once the PDA layer was formed, the layer-by-layer (LBL) technique was used to immobilize lipase. Cationic polyethylenimine (PEI) and anionic lipase were alternatively adsorbed on the PDA-modified surface for eight times. Compared with single layer adsorption, polyelectrolyte multilayer adsorption not only stabilized the enzyme, but also provided the network with a higher enzyme-loading capacity. The modification of PTFE microchannel and multilayer assembly were demonstrate by water contact angle measurement, Fourier transform infrared spectroscopy (FI-IR) and X-ray photoelectron spectroscopy (XPS). The morphological characteristics and biocompatible network were observed using Atomic Force Microscopy (AFM) and field emission scanning electron microscope (FE-SEM). The novelty of the work relies on the three points: (1) facile and easy modification of PTFE using moderate dopamine self-polymerization; (2) layer-by-layer method to form PEI/CAL-b network to increase enzyme loading and to stabilize enzyme; and (3) the efficiency of lipaseimmobilized microreactor was further tested in the bioproduction, more specifically, in the wax ester production, using oleic acid and long-chain alcohols (C10-C18) in the solvent-free system, which showed faster reaction speed and excellent stability.

\section{Materials and methods}

\subsection{Materials}

Candida antarctica lipase B was provided by Auwitkey Technology Ltd. co. (>10 $\mathrm{U} \mathrm{mg}^{-1}$ ). Polytetrafluoroethylene (PTFE) microtube (with inner diameter $0.38 \mathrm{~mm}$ and outer diameter $0.78 \mathrm{~mm}$ ) was purchased from Yue Yang Electronic Science and Technology Co. Ltd. Suzhou China. Dopamine hydrochloride was purchased from Sigma Aldrich. Polyethyleneimine (PEI) ( $M_{\mathrm{w}}=10 \mathrm{k}$, branched) was purchased from MICXY REAGENT. Oleic acid (85\%), citronellol (C10, 99\%), lauryl alcohol (C12, 99\%), tetradecyl alcohol (C14, 99\%), cetyl alcohol (C16, 99\%), and oleyl alcohol (85\%) were purchased from Aladdin Industrial Inc. All other reagents and solvents were of analytical grade. All the solutions were prepared with double-distilled water.

\subsection{Polydopamine coating of PTFE microchannel and enzyme immobilization}

The PTFE microchannel with desired length was first cut and cleaned by alcohol and ultrasound for $15 \mathrm{~min}$, and then successively washed with distilled water for $10 \mathrm{~min}$ to completely remove the residual alcohol. In order to form the polydopamine coating on the inner surface, the microchannel was filled with $2 \mathrm{mg} \mathrm{mL}{ }^{-1}$ dopamine in $0.1 \mathrm{M}$ Tris-HCl buffer solution ( $\mathrm{pH}$ 8.5) and the dopamine solution was kept injecting to the channel at a rate of $5 \mu \mathrm{L} \mathrm{min}{ }^{-1}$ by an injection pump for 12 hours. The purpose of continuous injection was to replace the oxidized dopamine with fresh solution. Once the polydopamine layer was formed, the microchannel was flushed with the Tris-HCl solution (0.1 M, pH 8.5) for further functionalization.

Enzyme immobilization procedure was then carried out using the layer by layer assembly. Both PEI and lipase were dissolved in the Tris-HCl buffer with pH 8.5 (50 mM). The PDAmodified microchannel was first filled with $10 \mathrm{mg} \mathrm{mL}^{-1}$ PEI solution and stayed for 1 hour to reach the adsorption equilibrium. The microchannel was washed with the Tris-HCl buffer to remove the weakly adsorbed PEI. $8 \mathrm{mg} \mathrm{mL}{ }^{-1}$ lipase solution was then injected to fill the microchannel and stayed for another 1 hour. After the microchannel was washed with Tris-HCl buffer again, the first layer (PEI/lipase) was completed. An 8-layer assembly was formed by repeating the two steps using different concentration of PEI and lipase. The concentration of PEI and lipase solution used for different layers was listed in Table 1. After alternative deposition for 8 layers, the microchannel immobilized with lipase was ready to use.

\subsection{Characterization of modified-PTFE surface}

As the inner diameter of PTFE microchannel was too small to cut open, we chose the PTFE membrane for characterization. The PDA-modified PTFE membrane was generated by dipping the membrane into dopamine solution for 12 hours, and the modified membrane was alternatively immersed in PEI and lipase solutions for an hour to prepare the multilayer assembly membrane for further characterization.

2.3.1 Attenuated total reflectance Fourier transform infrared (ATR-FTIR) measurement. The IR spectra of pristine PTFE, PDA-modified PTFE (PTFE-PDA), and the first layer of polyelectrolytes adsorption, including PEI adsorbed PTFE (PTFE-PDA-PEI) and lipase adsorbed PTFE (PTFE-PDA-PEIlipase), were acquired using a Nicolet iS50 instrument.

Table 1 Concentration of $\mathrm{PEI}$ and lipase involved in multilayer assembly

\begin{tabular}{lll}
\hline Layers & $\begin{array}{l}\text { Concentration } \\
\text { of } \mathrm{PEI}^{a}, \mathrm{mg} \mathrm{mL}\end{array}$ & $\begin{array}{l}\text { Concentration } \\
\text { of lipase solution }\end{array}$ \\
\hline 1 & 10 & $\mathrm{mg} \mathrm{mL}$ \\
2 & 10 & 8 \\
3 & 10 & 8 \\
4 & 10 & 8 \\
5 & 15 & 10 \\
6 & 15 & 10 \\
7 & 20 & 20 \\
8 & 20 & 20 \\
$a$ &
\end{tabular}


2.3.2 X-ray photoelectron spectroscopy (XPS) measurement. In order to verify the results of IR spectra, XPS measurements of PTFE, PTFE-PDA and multilayer-modified PTFE membranes are conducted using PHI 5000 VersaProbe (UlVAC-PHI company, Japan). The quantitative analysis of chemical composition of the coating layer was performed as well.

2.3.3 Measurement of the contact angles. The static-state water contact angles of native PTFEs and modified PTFE membranes were measured by POWEREACH contact angle measurement instrument. A droplet of water was dropped on the flat membrane, and the contact angle measurement is obtained in 20 seconds. The mean of the measurement was calculated from three measurements.

2.3.4 Characterization of morphology of modified PTFE. The morphology of PTFE before and after modification was determined by an Atomic Force Microscopy (AFM) and a field emission scanning electron microscope (FE-SEM). Specifically, the roughness and topography of the surface was determined by an atomic force microscope (Dimension ICON, Bruker AXS company) with resolution of $1 \mu \mathrm{m}$. Multilayer formed over the PTFE membrane was observed by an FE-SEM (Agilent 8500).

\subsection{Activity assay of immobilized lipase}

$P$-Nitrophenyl acetate ( $p$-NPA), was used as substrate to test the hydrolysis activity of the lipase and the assay was carried out at room temperature $\left(25{ }^{\circ} \mathrm{C}\right)$. The substrate solution was composed of $0.5 \mathrm{~mL}$ of acetonitrile containing $p$-nitrophenyl acetate ( $p$-NPA) $(0.05 \mathrm{M})$ and $3.5 \mathrm{~mL}$ of sodium phosphate buffer $(\mathrm{pH} 7.0,0.02 \mathrm{M})$. The freshly mixed solution was then injected into the $15 \mathrm{~cm}$ length microchannel at a flow rate of $8 \mu \mathrm{L} \mathrm{min} \mathrm{m}^{-1}$. After 3 minute's reaction, the effluent (hydrolysis products) was examined for the production of 4-NP using an UV-vis spectrophotometer by reading absorbance at $400 \mathrm{~nm}$. A calibration curve was obtained by measuring the absorbance of $p$-nitrophenol (pNP) standard solutions prepared in the same solvent mixture (acetonitrile : sodium phosphate buffer (1:7)). The concentration of pNP in effluent was then calculated and lipase enzyme activity was determined. The enzyme activity was expressed as amount of pNP formed on the microreactor under the condition used per minute. One enzyme unit (U) was the amount of protein liberating $1 \mu \mathrm{g}$ of pNP per minute.

\subsection{Determination of lipase loading on different layers in microreactor}

The amount of lipase immobilized on the inner surface of microchannel was determined by calculating the difference between the amount of lipase in the initial loading solution and that in the residual solution collected after the adsorption process. Bradford assay was used here to confirm the protein in solution.

\subsection{Production of wax ester using PTFE microreactor}

In order to verify the feasibility of our approach, esterification between oleic acid and long-chain alcohols is performed in the micro-reactor. The long-chain alcohols used in this experiment including citronellol (C10), lauryl alcohol (C12), tetradecyl alcohol (C14), cetyl alcohol (C16) and oleyl alcohol (C18 : 1). A 5 meter microreactor was used, and an appropriate amount of acid and alcohol were thoroughly mixed and injected into the microreactor at a rate of $16 \mu \mathrm{L} \mathrm{min}{ }^{-1}$. Under such condition, a 25 minute residence time (reaction time) was set. Samples were collected periodically from the microchannel outlet and

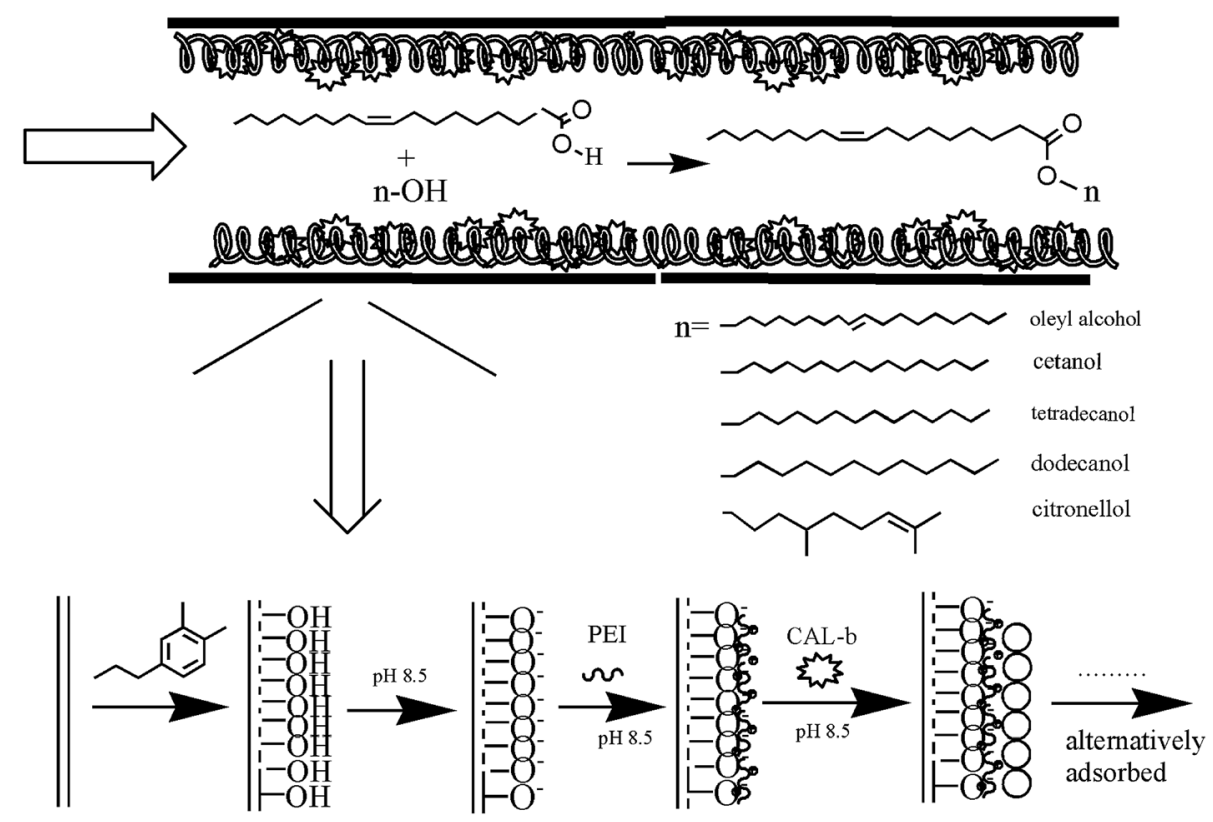

Fig. 1 Process of immobilization of CAL-b based on self-oxidation of dopamine and LBL method. Long chains with positive charges represented $\mathrm{PEI}$, and the circles represented the lipase. 


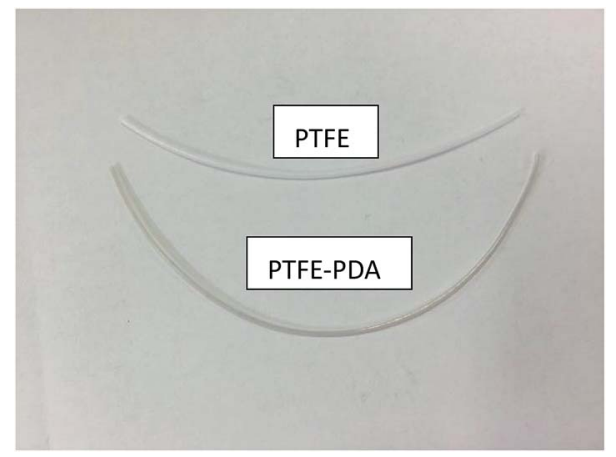

Fig. 2 PTFE microchannel before and after PDA modification.

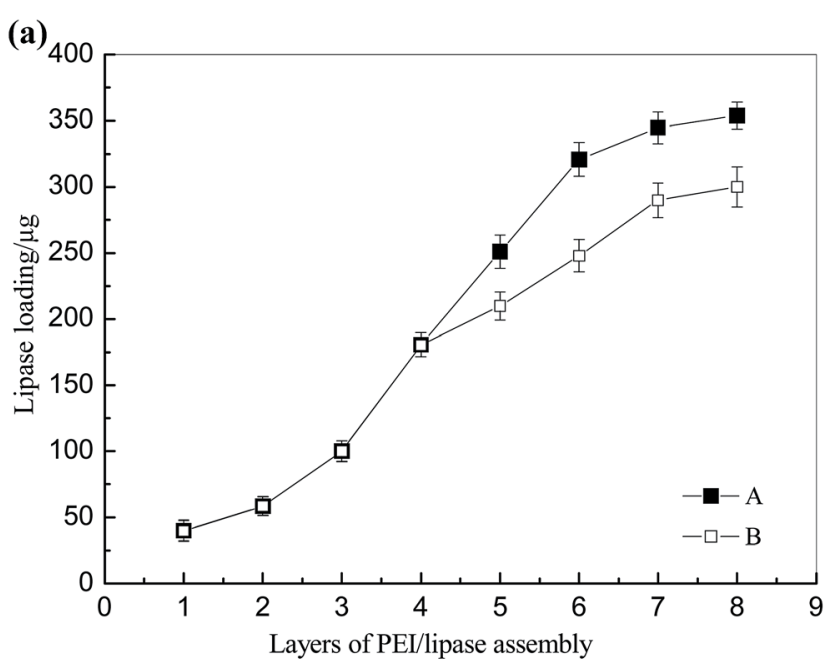

(b)

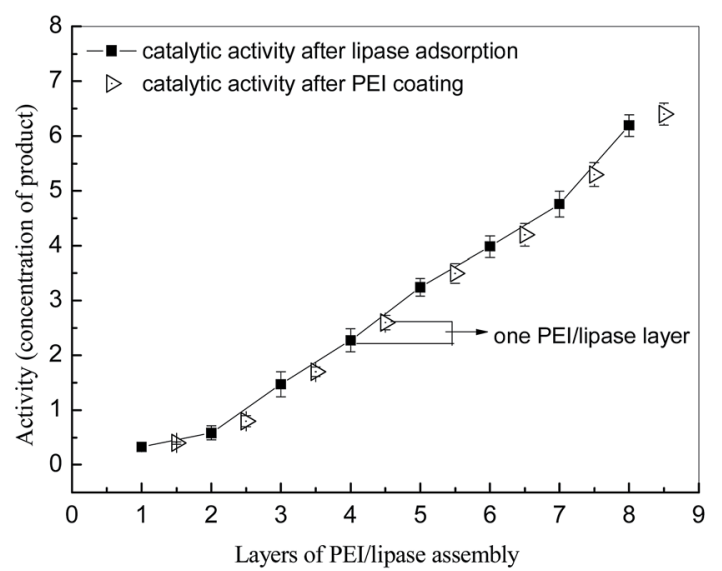

Fig. 3 (a) Effect of the number of layer on lipase loading. A $5 \mathrm{~m}$ microchannel was used to immobilize the enzyme. Line A represents the lipase loading on different layers under the condition of improved concentration of PEI and lipase after 4 layers; line B represents the lipase loading on different layers with the same concentration of $\mathrm{PEI}$ and lipase keep the same during the 8-layer assembly. (b) Catalytic activity of lipase adsorption and PEI coating during multilayerassembly. Black square represents the catalytic activity of microreactors after the lipase adsorption, while the scattered white triangles on the right side of the black square represents the catalytic activity after the PEI coating in corresponding layers. passed through $0.22 \mu \mathrm{m}$ filters for gas chromatography (GC) test.

\subsection{Identification of the reaction product}

Reactants were quantitated by injecting $1 \mu \mathrm{L}$ diluted samples into a gas chromatographer (SP-7890, ShanDong, China) equipped with a flame-ionization detector (FID) and DB-225 column $(30 \mathrm{~m} \times \mathrm{mm} \times 0.10 \mu \mathrm{m}$; Agilent $)$. FID and injection temperature were set to $300{ }^{\circ} \mathrm{C}$ and $320{ }^{\circ} \mathrm{C}$, respectively. The oven temperature was maintained at $160{ }^{\circ} \mathrm{C}$ for $1 \mathrm{~min}$, followed by elevation to $220{ }^{\circ} \mathrm{C}$ at a rate of $15^{\circ} \mathrm{C} \mathrm{min}{ }^{-1}$. After that, it was further increased to $300{ }^{\circ} \mathrm{C}$ at a rate of $20^{\circ} \mathrm{C} \mathrm{min}{ }^{-1}$, and stayed for $3.8 \mathrm{~min}$. Nitrogen was used as the carrier gas. Octodecane was used as an internal standard. The yield of the reaction was quantified based on the ratio of consumed oleic acid to the total amount of oleic acid before the reaction.

\section{Results and discussion}

\subsection{Preparation of PTFE microreactor by polydopamine- mediated multilayer assembly}

The design of enzyme-immobilizing method in microchannel is the key step in preparing enzymatic-immobilized microreactors. In our study, the PTFE open-tubular EI-MR was developed in a facile and convenient way. The modification process of PTFE was shown in Fig. 1. Firstly, a PDA layer was formed on the inner surface of the PTFE microchannel from the self-polymerization of dopamine. As shown in Fig. 2, the semitransparent PTFE microchannel underwent color change and turned into grey caused by the dark PDA layer formed in the inner surface of the channel after 12 hour oxidative modification. Increasing the time to more than 12 hours had no influence on the following steps (data not shown). Under $\mathrm{pH} 8.5$, PDA-modified PTFE surface would carry negative charges. ${ }^{22}$

The second step was to form a multilayer assembly using PEI and lipase. Under the alkaline condition, PEI with a large

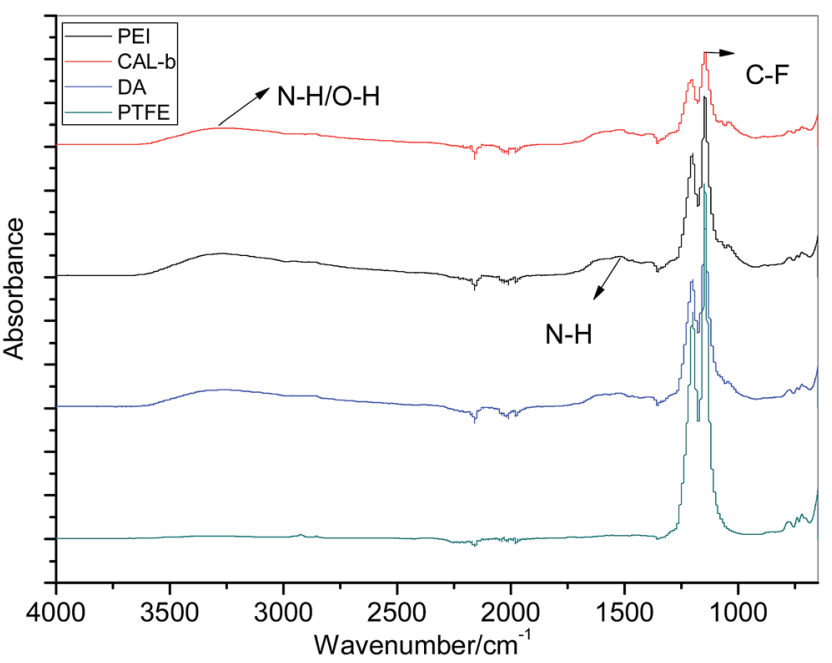

Fig. 4 FTIR spectra of PTFE, PDA-modified PTFE, PEI-deposited PTFE and lipase-deposited PTFE. 

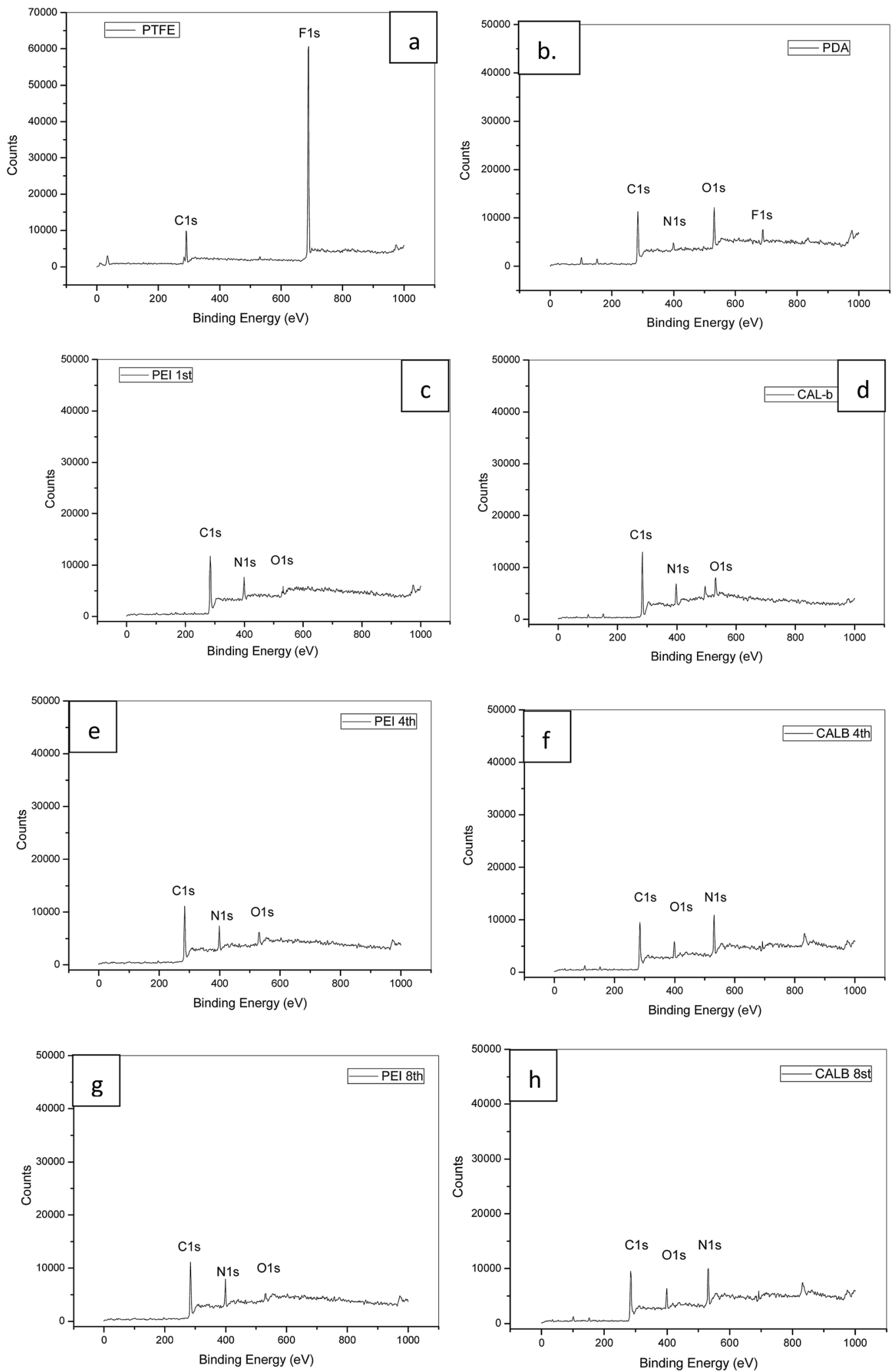

Fig. 5 (a-h) XPS spectra for PTFE, PDA-modified PTFE, PEI and lipase deposited PTFE membranes.

number of amino groups can still protonize and provide positive charges, which can adsorb on the PDA-modified PTFE microchannel. Meanwhile, under the same condition, lipase with negative charges ( $\mathrm{pI}<\mathrm{pH}$ ) will be immobilized on the surface under the electrostatic interaction. One PEI-lipase layer assembly on a 5 meter PDA-modified PTFE microchannel 
Table 2 Chemical composition of unmodified and modified PTFE surface

\begin{tabular}{|c|c|c|c|c|c|c|c|c|}
\hline & PTFE & PTFE-PDA & $\begin{array}{l}\text { PEI adsorption } \\
\text { in the 1st layer }\end{array}$ & $\begin{array}{l}\text { Lipase adsorption } \\
\text { in the 1st layer }\end{array}$ & $\begin{array}{l}\text { PEI adsorption } \\
\text { in the } 4 \text { th layer }\end{array}$ & $\begin{array}{l}\text { Lipase adsorption } \\
\text { in the } 4 \text { th layer }\end{array}$ & $\begin{array}{l}\text { PEI adsorption } \\
\text { in the 8th layer }\end{array}$ & $\begin{array}{l}\text { Lipase adsorption } \\
\text { in the 8th layer }\end{array}$ \\
\hline $\mathrm{C}$ & 34.82 & 68.35 & 67.89 & 70.18 & 66.31 & 71.81 & 67.98 & 71.23 \\
\hline $\mathrm{O}$ & - & 20.22 & 2.62 & 18.94 & 1.53 & 18.23 & 2.08 & 17.56 \\
\hline F & 65.18 & 3.65 & - & - & - & - & - & - \\
\hline $\mathrm{N} / \mathrm{C}$ & - & 0.113 & 0.43 & 0.155 & 0.483 & 0.138 & 0.44 & 0.157 \\
\hline
\end{tabular}

Table 3 Contact angles of unmodified and modified PTFE surface

\begin{tabular}{lllll}
\hline & PTFE & DA & PEI & CAL-b \\
\hline Contact angle & $105^{\circ} \pm 3.1^{\circ}$ & $46.5^{\circ} \pm 5.2^{\circ}$ & $20.5^{\circ} \pm 2.3^{\circ}$ & $11^{\circ} \pm 3.4^{\circ}$
\end{tabular}

resulted in approximately $25 \mu \mathrm{g}$ lipase immobilization, which was not enough for biosynthesis. So it is crucial to establish a multilayer by alternatively depositing PEI and lipase onto the microchannel. It was indicated in Fig. 3(a) that amount of lipase immobilization was in direct proportion to the number of layer till it reached to the plateau in layer 8 (approximately $350 \mu \mathrm{g}$ to $400 \mu \mathrm{g} / 5 \mathrm{~m}$ microchannel). Multilayer assembly has been applied in researches to immobilize different kinds of enzymes. ${ }^{23-26}$ Liu prepared multilayer-assemble microchip using chitosan (CS, polycation) and hyaluronic acid (HA, polyanion). ${ }^{23,24}$ Trypsin was then imbedding the multilayer and stabilized by hydrogen bonding force. Poly(diallyldimethylammonium chloride) (PDDA) and graphene oxide (GO) were also used as multilayer "cushion" for enzyme immobilization. ${ }^{26}$ Comparing with existing study, our approach benefited by the lipase used in our study, which was functionalized as polyanion. It brought two advantages: (1) enzyme loading got increased in our approach since the enzyme was immobilized on the surface by not only hydrogen bonds, but also electrostatic interactions. An enzyme loading of $107 \mathrm{mg} \mathrm{m}^{-2}$ has been reported in Liu's work using four times of CS-HA-CS-HA-CS deposition, while only eight-layers of PEI-lipase correspond to an enzyme loading of $252 \mathrm{mg} \mathrm{m}^{-2}$ in our study, and it was more than doubled Liu's results; (2) the improvement of the enzyme activity was observed as lipase was embedded in PEI sandwich structures. Multilayers with abundant functional groups provided biocompatible microenvironment to immobilized enzymes. Moreover, it was reported that the PEI coating can be used to stabilize and stimulate enzyme activity. ${ }^{27,28}$ As shown in Fig. 3(b), the improvement of activity after PEI coating was confirmed in our study as well. We conjectured that the PEI coating may have some positive effects on the "lid" structure of lipase and prevent the oxidation of proteins. However, more in-depth researches should be done in further study.

\subsection{Characterization of modified PTFE microchannel}

In order to verify the formation of polydopamine layer and PEI/ lipase assembly, several characterization methods were used to characterize the modified PTFE microchannel. ATR-FTIR spectral analysis and XPS were used to characterize the chemical composition of PTFE surface before and after deposition. As shown in Fig. 4, unmodified PTFE surface showed an intense absorption in around $1200 \mathrm{~cm}^{-1}$, which was attributed to C-F bond. ${ }^{29}$ Compared with the pristine PTFE, a new broad absorption between $3500 \mathrm{~cm}^{-1}$ and $3100 \mathrm{~cm}^{-1}$ was observed for the PTFEPDA sample, which was assigned to $\mathrm{N}-\mathrm{H} / \mathrm{O}-\mathrm{H}$ stretching vibrations. Another two new peaks at $1600 \mathrm{~cm}^{-1}$ and $1509 \mathrm{~cm}^{-1}$ indicated the overlapping of $\mathrm{C}=\mathrm{C}$ resonance vibration in the aromatic ring in the PDA layer and the $\mathrm{N}-\mathrm{H}$ bending vibration. ${ }^{30}$ After the deposition of PEI and lipase, absorption intensity of $3500 \mathrm{~cm}^{-1}$ to $3100 \mathrm{~cm}^{-1}$, and $1509 \mathrm{~cm}^{-1}$ kept increasing, which was due to the absorption of the amino groups on PEI and lipase. Meanwhile, the absorption in $1200 \mathrm{~cm}^{-1}$ continued to decrease, indicating the PEI coatings and protein layers successfully deposited.

The quantification of atomic composition by XPS further confirmed the speculation in FTIR. After the PDA coating, new peaks for nitrogen (N 1s) and oxygen (O 1s) appeared, while fluorine (F 1s) got dramatically weakened (Fig. 5(b)). As shown in Table 2, the N/C ratio for the layer was 0.113 (Table 2), which was closed to the theoretical value (0.125) of dopamine. ${ }^{31}$ In order to confirm the successful deposition of PEI and lipase in

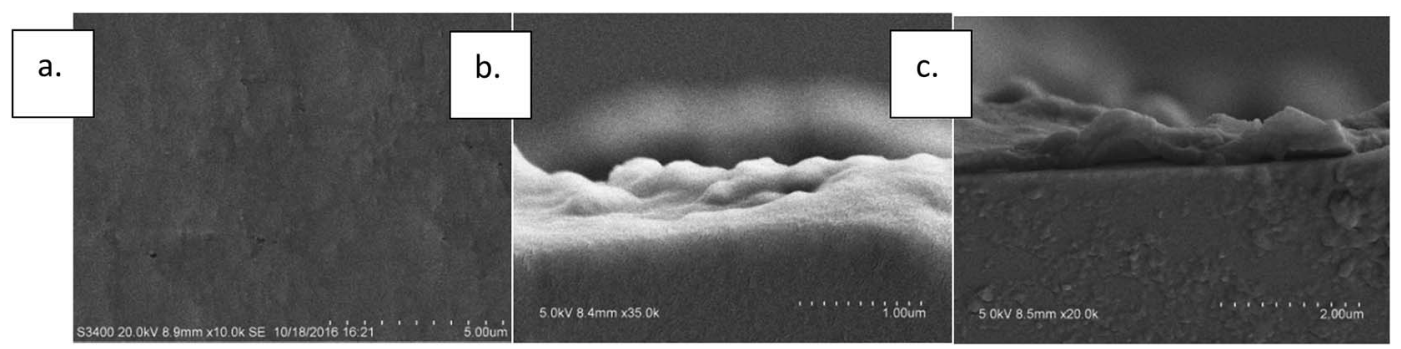

Fig. 6 FESEM photographs of unmodified and modified PTFE surface: (a) unmodified PTFE surface; (b) PTFE surface after PDA modification and PEI/lipase assembly; (c) cross-section photograph of PTFE surface after PDA modification and PEI/lipase assembly. 
different layers, chemical compositions on the $1^{\text {st }}, 4^{\text {th }}$ and $8^{\text {th }}$ layer were also tested. It turned out that N/C ratios were around 0.44 for PEI depositions, and decreased to around 0.155 after lipase adsorptions in the tested layers, which confirmed the alternatively deposition of PEI and lipase. The oxygen (O 1s) adsorption in PEI adsorbed layer was considered as the signal of underlying layers (Fig. 5(c, e and g)). Contact angles during the multilayer assembly process were monitored as well. As shown in Table 3, the hydrophobic PTFE surface with contact angle of $105^{\circ}$ was covered by the hydrophilic PDA layer with contact angle of $46.5^{\circ}$, which was in accordance with other researches. ${ }^{32}$ After the PEI adsorption, the contact angle kept decreasing to around $20^{\circ}$, and settled to $11^{\circ}$ when lipase was deposited. The results indicated that enzyme was immobilized in hydrophilic microchannel, which benefited the enzyme activity. Combined the results from FTIR spectra, XPS spectra, and contact angle changes, the progressive assembly of PDA and PEI/lipase assembly was monitored and confirmed.

Morphology changes can also be observed in SEM photos. It can be seen in Fig. 6(a) and (b) that unmodified PTFE membrane was smooth with a few wrinkles. On the other hand, the surface of the membrane became rough and fluctuated up and down after the PDA modification and multilayers piled up. A clear evidence of polymer layer formation can be seen in the profile view shown in Fig. 6(c).

$\mathrm{Ra}$ (arithmetical mean roughness) values and threedimensional images of PTFE surface were recorded by the atomic force microscopy (AFM) as shown in Table 4 and Fig. 7(a-d). The roughness of PTFE surface was $24.2 \mathrm{~nm}$, and increased as the PDA modification and PEI adsorption proceeded. The lipase adsorption reduced the surface roughness, which may be the result of enzyme incorporation in the polyelectrolyte network. 3D images of surfaces confirmed the speculation. Fig. $7(\mathrm{a}-\mathrm{d})$ clearly showed the surface change as the modification proceeded.

\subsection{Production of wax ester using the microreactor}

Liquid wax esters are valuable organics and have been widely used in cosmetic, lubricant, or food industries due to their nontoxic characteristics and excellent wetting behavior in

Table 4 Change of roughness during the modification process

\begin{tabular}{lllll}
\hline & PTFE & PTFE-PDA & PEI & CAL-b \\
\hline Roughness & $24.2 \pm 1.3 \mathrm{~nm}$ & $35.8 \pm 2.1 \mathrm{~nm}$ & $71.3 \pm 3.5 \mathrm{~nm}$
\end{tabular}

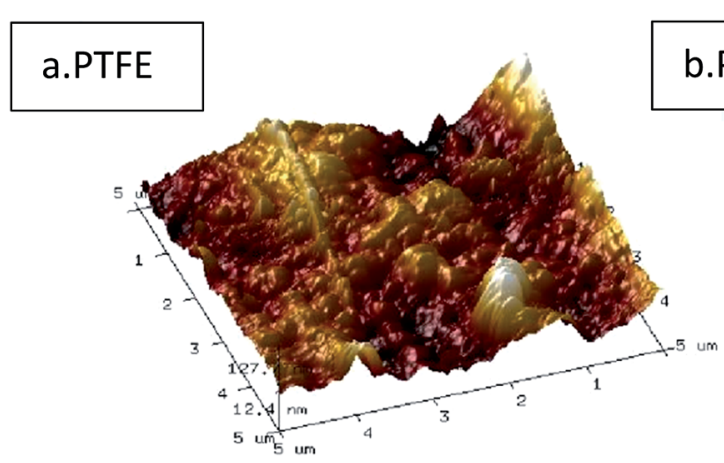

\section{b.PTFE-PDA}
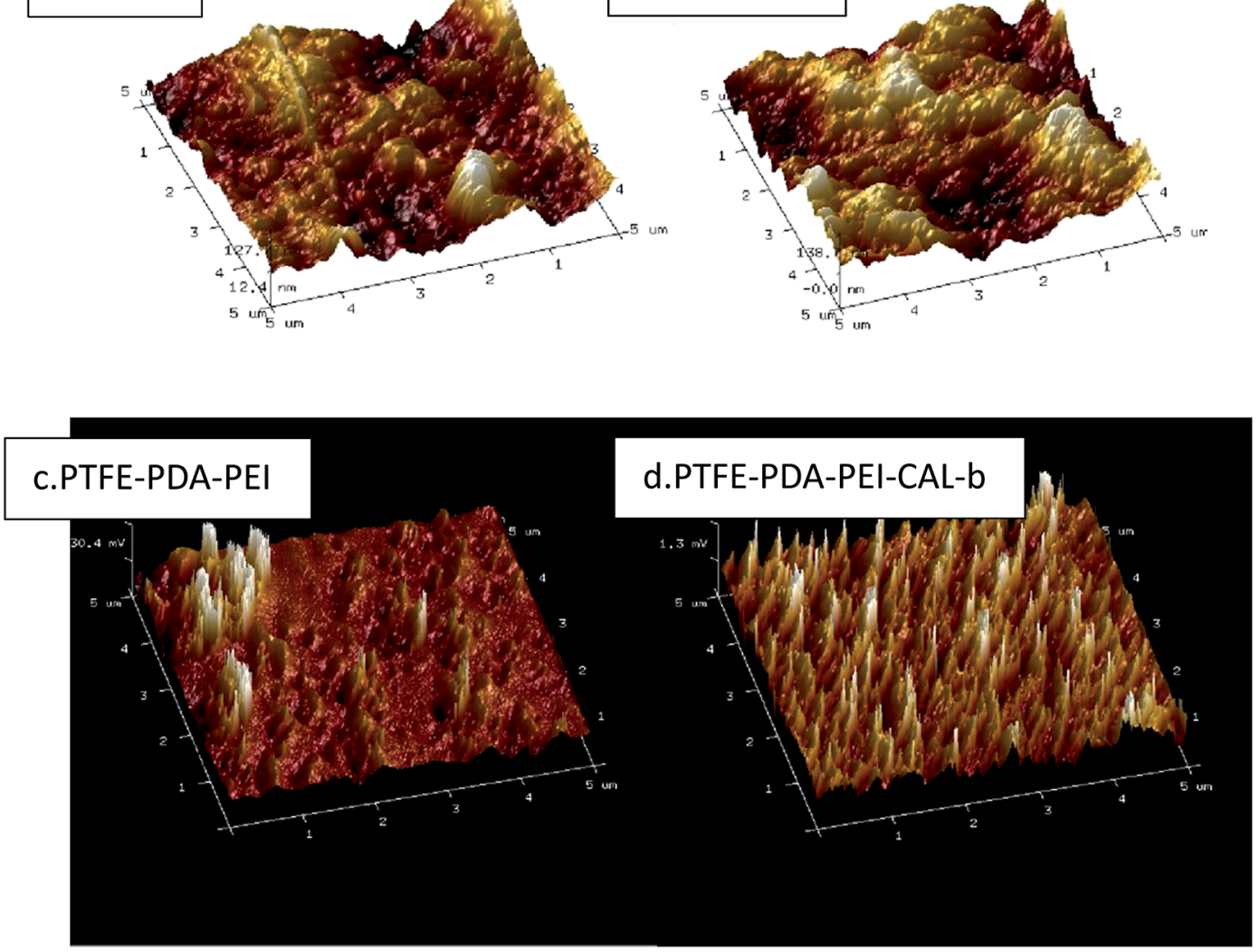

Fig. 7 (a-d) AFM photographs of modified and unmodified PTFE surface. 


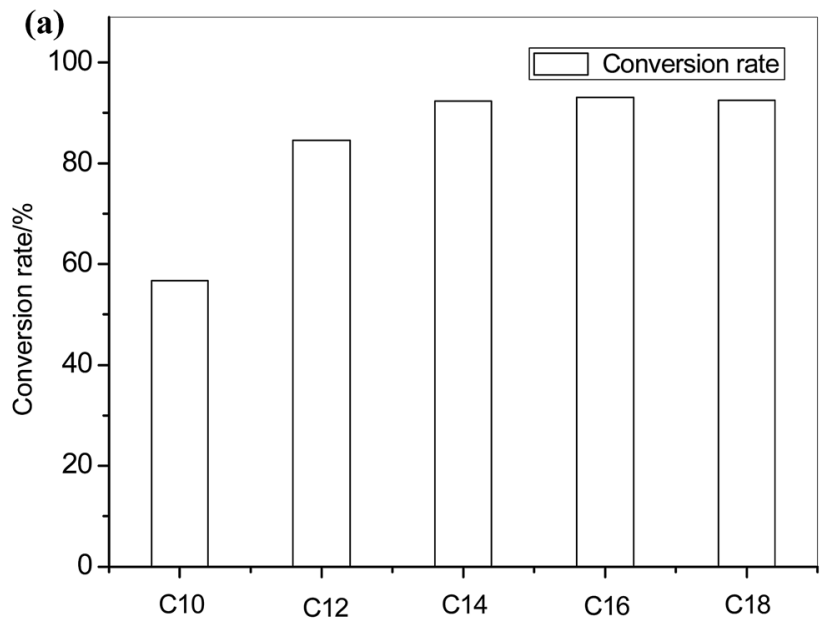

(b)

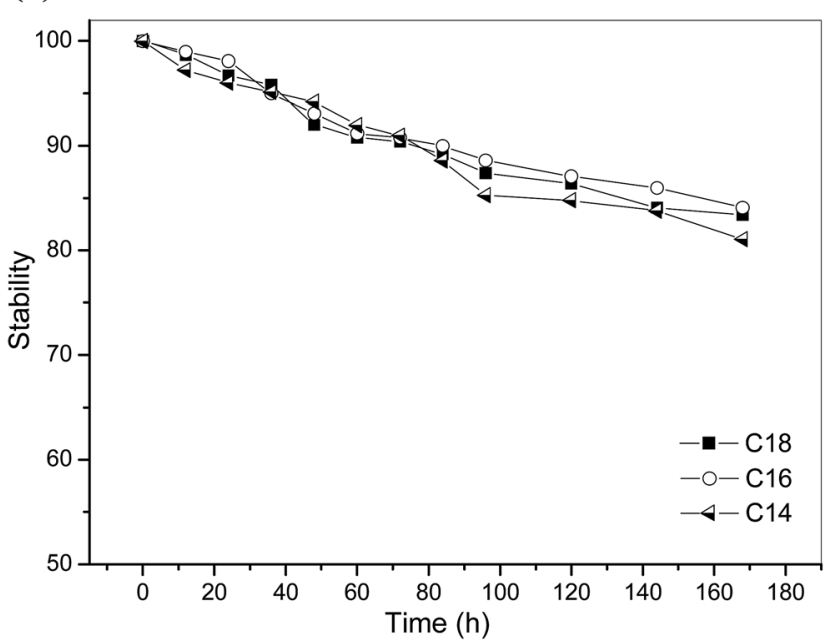

Fig. 8 (a) Conversion rate of different oleates under the same reaction conditions. Setup: molar ratio: $1: 1$, length of microreactor: $5 \mathrm{~m}$, temperature: $40{ }^{\circ} \mathrm{C}$, reaction time: $25 \mathrm{~min}$. (b) Reusability of microreactor on esterification of oleate. Setup: molar ratio: $1: 1$, length of microreactor: $5 \mathrm{~m}$, temperature: $40^{\circ} \mathrm{C}$, reaction time: $25 \mathrm{~min}$.

interfaces. ${ }^{33}$ Wax ester can be produced by esterification reaction between long chain alcohols and long chain acids. Esterification of oleic acid and long chain alcohols were performed using the open-tubular EI-MR in our study.

The conversion rate of oleic acid and different long chain alcohols (C10-C18) was shown in Fig. 8. Except for citronellol (C10), other alcohols successfully reacted with oleic acid and reached over $80 \%$ conversion rate in 25 minutes. The lower conversion rate between citronellol (C10) and oleic acid was probably due to the selectivity of CAL-b on the long-chain alcohol. Compared with traditional reactors, the reaction efficiency of the wax ester production was at least two times improved using microreactor, ${ }^{34}$ and almost 8 times faster than commercial Novozyme 435 in our previous test ( 4 hours to reach $85 \%$ conversion rate). The microreactor also exhibited excellent stability. It was shown in Fig. 8(b) that over $80 \%$ conversion rate can be maintained in 7 days for the esterification between oleic acid and tetradecyl alcohol (C14), cetyl alcohol (C16) and oleyl alcohol (C18: 1).

\section{Conclusion}

An easy and facile way was proposed to form an open-tubular EIMR using PTFE microchannel. The lipase was immobilized on the polydopamine-modified PTFE microchannel using the LBL method. The formation of PDA layer and the multilayer deposition process was monitored by various methods, and photographs of FE-SEM and AFM kept track of the changes of morphology. Our approach has two major advantages over existing methods: the doubled enzyme loading $\left(252 \mathrm{mg} \mathrm{m}^{-2}\right)$ and the improvement of the enzyme stability. The open-tubular EI-MR has been applied in the production of the wax ester and the production efficiency was considerably improved. The method has the potential for a broader application.

\section{Acknowledgements}

This work was supported by the Major State Basic Research Development Program of China (973 21 Program) (Grant No. 2013CB733904), and National Natural Science Foundation of China (Grant 22 No. 21406114).

\section{References}

1 F. J. Contesini, D. B. Lope, G. A. Macedo, et al., Aspergillus sp. lipase: potential biocatalyst for industrial use, J. Mol. Catal. B: Enzym., 2010, 67, 163-171.

2 P. Ye, Z. K. Xu, A. F. Che, J. Wu and P. Seta, Chitosan-tethered poly(acrylonitrile-co-maleic acid) hollow fiber membrane for lipase immobilization, Biomaterials, 2005, 26, 6394-6403.

3 Y. Asanomi, H. Yamaguchi, M. Miyazaki, et al., EnzymeImmobilized Microfluidic Process Reactors, Molecules, 2011, 16, 6041-6059.

4 J. Kaneno, R. Kohama, M. Miyazaki, M. Uehara, et al., A simple method for surface modification of microchannels, New J. Chem., 2003, 27, 1765-1768.

5 S. Matsuura, R. Ishii, T. Itoh, S. Hamakawa, et al., Immobilization of enzyme-encapsulated nanoporous material in a microreactor and reaction analysis, Chem. Eng. J., 2011, 167, 744-749.

6 K. Kawakami, Y. Sera, S. Sakai, et al., Development and Characterization of a Silica Monolith Immobilized Enzyme Micro-bioreactor, Ind. Eng. Chem. Res., 2005, 44, 236-240.

7 P. He, G. Greenway and S. J. Haswell, Development of a monolith based immobilized lipase micro-reactor for biocatalytic reactions in a biphasic mobile system, Process Biochem., 2010, 45, 593-597.

8 A. S. Bhangale, K. L. Beers and R. A. Gross, Enzyme-Catalyzed Polymerization of End-Functionalized Polymers in a Microreactor, Macromolecules, 2012, 45, 7000-7008.

9 S. Matosevic, N. Szita and F. Baganz, Fundamentals and applications of immobilized microfluidic enzymatic reactors, J. Chem. Technol. Biotechnol., 2011, 86, 325-334.

10 M. Z. Gan, J. Su, J. Wang, et al., A scalable microfluidic chip for bacterial suspension culture, Lab Chip, 2011, 11, 4087. 
11 H. B. Mao, T. L. Yang and P. S. Cremer, Design and Characterization of Immobilized Enzymes in Microfluidic Systems, Anal. Chem., 2002, 74, 379-385.

12 Y. Li, X. Q. Xu, B. Yan, et al., Microchip Reactor Packed with Metal-Ion Chelated Magnetic Silica Microspheres for Highly Efficient Proteolysis, J. Proteome Res., 2007, 6, 2367-2375.

13 S. Kataoka, Y. Takeuchi, A. Harada, et al., Microreactor with mesoporous silica support layer for lipase catalyzed enantioselective transesterification, Green Chem., 2010, 12, 331-337.

14 G. Ghafourifar and K. C. Waldron, Fluorescence Microscopy Imaging of an Immobilized Enzyme Microreactor to Investigate Glutaraldehyde-Mediated Crosslinking of Chymotrypsin, Anal. Lett., 2016, 49, 589-600.

15 Y. Asanomi, H. Yamaguchi, M. Miyazaki, et al., EnzymeImmobilized Microfluidic Process Reactors, Molecules, 2011, 16, 6041-6059.

16 F. Chen, S. Liu, J. Y. Liu, et al., Surface modification of tube inner wall by transferred atmospheric pressure plasma, Appl. Surf. Sci., 2016, 389, 967-976.

17 R. Takata, T. Iwao and M. Yumoto, Surface Modification of PTFE Using Low-Energy Nitrogen Ion Irradiation: Improvement in Adhesive Strength on Modification of Deep Modifying Layer, IEEJ Transactions on Fundamentals and Materials, 99, 2016, 93-99.

18 S. Okuji, H. Kitazawa and Y. Takeda, In situ analysis of ioninduced polymer surface modification using secondary ion mass spectroscopy, Nucl. Instrum. Methods Phys. Res., 2016, 377, 83-88.

19 H. Lee, S. M. Dellatore, W. M. Miller and P. B. Messersmith, Mussel-Inspired Surface Chemistry for Multifunctional Coatings, Science, 318, 2007, 426-430.

20 R. F. Luo, L. L. Tang, J. Wang, et al., Improved immobilization of biomolecules to quinone-rich polydopamine for efficient surface functionalization, Colloids Surf., B, 2013, 106, 66-73.

21 X. B. Yin and D. Y. Liu, Polydopamine-based permanent coating capillary electrochromatography for auxin determination, J. Chromatogr. A, 2008, 1212, 130-136.

22 H. L. Wei, J. Ren, B. Han, et al., Stability of polydopamine and poly(DOPA) melanin-like films on the surface of polymer membranes under strongly acidic and alkaline conditions, Colloids Surf., B, 2013, 110, 22-28.

23 Y. Liu, H. J. Lu, W. Zhong, et al., Multilayer-Assembled Microchip for Enzyme Immobilization as Reactor Toward
Low-Level Protein Identification, Anal. Chem., 2006, 78, 801-808.

24 Y. Liu, W. Zhong, S. Meng, et al., Assembly-Controlled Biocompatible Interface on a Microchip: Strategy to Highly Efficient Proteolysis, Chem.-Eur. J., 2006, 12, 6585-6591.

25 Z. M. Tang, T. D. Wang and J. W. Kang, Immobilized capillary enzyme reactor based on layer-by-layer assembling acetylcholinesterase for inhibitor screening by CE, Electrophoresis, 2007, 28, 2981-2987.

26 Z. R. Yin, W. W. Zhao, M. M. Tian, et al., A capillary electrophoresis-based immobilized enzyme reactor using graphene oxide as a support via layer by layer electrostatic assembly, Analyst, 2014, 139, 1973-1979.

27 M. M. Andersson, J. D. Breccia and H. K. Rajni, Stabilizing effect of chemical additives against oxidation of lactate dehydrogenase, Biotechnol. Appl. Biochem., 2000, 32, 145153.

28 M. P. Sonia, F. Marco, M. G. Jose, et al., Synthesis of ascorbyl oleate by transesterification of olive oil with ascorbic acid in polar organic media catalyzed by immobilized lipases, Chem. Phys. Lipids, 2013, 174, 48-54.

29 A. Shojaei and S. Gholamalipour, Effect of Chemical Treatment of Teflon Powder on the Properties of Polyamide 66/Teflon Composites Prepared by Melt Mixing, Macromol. Res., 2011, 19, 613-621.

30 Z. Y. Xi, Y. Y. Xu, L. P. Zhu, et al., A facile method of surface modification for hydrophobic polymer membranes based on the adhesive behavior of poly(DOPA) and poly(dopamine), $J$. Membr. Sci., 2009, 327, 244-253.

31 Q. Wei, F. L. Zhang, J. Li, et al., Oxidant-induced dopamine polymerization for multifunctional coatings, Polym. Chem., 2010, 1, 1430-1433.

32 L. Jiang, G. C. Jin, J. Y. Kang, et al., Surface Characteristics of Mussel-inspired Polydopamine Coating, J. Wuhan Univ. Technol., Mater. Sci. Ed., 2014, 29, 197-200.

33 Y. Isano, M. Nakajima and H. Nabetani, Solvent-free esterification of oleic acid and oleyl alcohol using membrane reactor and lipase-surfactant complex, $J$. Ferment. Bioeng., 1996, 86, 138-140.

34 S. M. Radzi, M. Basri, A. B. Salleh, et al., Optimization study of large-scale enzymatic synthesis of oleyl oleate, a liquid wax ester, by response surface methodology, J. Chem. Technol. Biotechnol., 2006, 81, 374-380. 